\title{
Evaluación del Bosque Seco Tropical del Parque Histórico Guayaquil
}

\section{Evaluation of the Flood Plain Forest of the Guayaquil Historical Park}

Juan Carlos Cisneros Bohórquez ${ }^{a}$, Natalia Molina Moreira ${ }^{b}$

\author{
INFORMACIÓN DEL \\ ARTÍCULO \\ Fecha de recepción:26 de agosto del \\ 2016 \\ Fecha de aceptación: 15 de marzo del \\ 2017
}

\footnotetext{
$a$ Ingeniero en Gestión Ambiental, Universidad Espíritu Santo - Ecuador. E-mail: juancisneros@uees.edu.ec
}

\footnotetext{
${ }^{b}$ Magíster en Ciencias en Agricultura Tropical Sostenible. Bióloga. Docente investigadora, Universidad Espíritu Santo - Ecuador.

E-mail: natimolina@uees.edu.ec
}

\begin{abstract}
Resumen
El Parque Histórico Guayaquil mantiene, en su Zona de Vida Silvestre, un Bosque Seco Tropical establecido mediante el Proyecto de Zonificación del Bosque desde el año 2002. A pesar de su importancia, no se han realizado inventarios forestales o evaluado el cumplimiento del Plan de Manejo Ambiental desde su implementación. Por este motivo se realizó un inventario de los árboles de este bosque, se registraron las medidas dasométricas, se elaboró un catálogo y se propusieron lineamientos para mejorar el Plan de Manejo. Se identificaron 378 individuos, pertenecientes a 62 especies y 26 familias, ilustradas en el catálogo. La mayoría se encuentran entre los 4 a $19 \mathrm{~cm}$ de diámetro; 3 a $9 \mathrm{~m}$ de altura y 2 a 4 $\mathrm{m}$ de copa, parámetros que corresponden a un bosque joven de crecimiento rápido en altura. Es necesario implementar lineamientos para el registro secuencial de información de este bosque para su conservación.
\end{abstract}

Palabras Clave:

Catálogo, dasometría, inventario forestal, lineamientos, crecimiento, especies.

Clasificación JEL: F18, N56.

\begin{abstract}
Parque Histórico Guayaquil maintains since 2002, in its Wildlife Zone, a Tropical Dry Forest established with the Forest Zonification Project. Despite its importance, no forest inventories or evaluation of the compliance of its Environmental Management Plan have been made since its implementation. For this reason, a forest inventory of all the species was elaborated; dasometric measurements were registered, a catalogue put together, and guidelines to improve the Management Plan were established. 378 individuals were identified, from 62 species and 26 families, illustrated in the catalogue. Most are between 4 and $19 \mathrm{~cm}$ of diameter, 3 to $9 \mathrm{~m}$ of height and 2 to $4 \mathrm{~m}$ of crown, parameters that correspond to a young forest of fast height growth. It is necessary to establish guidelines for the sequenced register of information of the forest conservation.
\end{abstract}

Keywords

Catalogue, dasometry, forest inventory, growth, guidelines species.

JEL Classification: F18, N56.

INVESTIGATIO No. 9, marzo 2017, pp. 1-27

(C) Universidad Espíritu Santo - UEES

ISSN: 1390 - 6399 


\section{Introducción}

Un antiguo proverbio indio dice: "la Tierra no es una herencia de nuestros padres, sino un préstamo de nuestros hijos". Por tanto, su conservación y preservación debería ser una responsabilidad adquirida de todos los sectores de la sociedad, con nuestras futuras generaciones. Contribuyendo con este objetivo se encuentra una de los más emblemáticos lugares turísticos del país: el Parque Histórico Guayaquil (PHG), "un parque temático cuyo objetivo es brindar un espacio de recreación y esparcimiento a visitantes o turistas de todas las edades" (Idrovo et. al., 2010), recreando los ecosistemas característicos de la Antigua Provincia de Guayaquil. Dentro de sus objetivos medioambientales, tiene como propósito restaurar bosques encontrados en la región litoral del Ecuador (Mendoza \& Molina-Moreira, 2015), como el Bosque Seco Tropical (BsT). Por este motivo en el año 2002 se aprobó el Proyecto de Zonificación del Bosque de la Zona Silvestre del Parque Histórico Guayaquil, en el que se propuso constituir bosques de la costa como manglar, garúa, llanura inundable y el bosque seco (Molina \& Molina, 2005).

Antes de la implementación del Proyecto de Zonificación del Bosque, en la Zona de Vida Silvestre (ZVS) se presentaba un bosque natural con "árboles de Triplaris cumingiana, Pithecellobium daulense, Terminalia cattapa, Mutingia calabura; Guazuma ulmifolia y arbustos espinosos de la familia MIMOSACEAE, entre otros". Para garantizar las condiciones favorables para el creci- miento de las especies características del BsT, "se elevó un promedio $60 \mathrm{~cm}$ del nivel del suelo con el lodo evacuado del manglar para forestar estas áreas con los cuatro estratos vegetales" con plantas provenientes de los viveros: Cerro Blanco, Jardín Botánico de Guayaquil, ESPOL y se colectaron plantas herbáceas y arbustivas en la vía a salinas, Bucay, vía Samborondón y en Tarifa (Molina \& Molina, 2005).

El PHG, como espacio cultural y medioambiental, contribuye a la conservación de especies vegetales de gran importancia para la memoria histórica de la Antigua Provincia de Guayaquil. Dentro de estas se encuentran especies vegetales características de los BsT, uno de los ecosistemas más amenazados por siglos, debido a su proximidad con zonas urbanas. Actualmente enfrentan una nueva amenaza por el cambio climático, por la posible alteración de los patrones de precipitación en las regiones de BsT (Calvo-Alvarado et al., 2013; Jaramillo et al., 2011).

Las especies vegetales son $35 \%$ nativas de la costa, $13 \%$ endémicas de la costa, $8 \%$ de otros lugares y $4 \%$ indeterminadas (Molina \& Molina, 2005). Sin embargo, a pesar de la importancia de la restauración ecológica de estos ecosistemas efectuada en el PHG, no se ha realizado un levantamiento constante de información para evaluar el establecimiento del bosque seco y la implementación del Plan de Manejo Ambiental (PMA). Por lo expuesto se actualizó el inventario de especies leñosas del BsT, se elaboró un catálogo con las especies identificadas, y 
se propusieron nuevos lineamientos para el PMA del Proyecto de Zonificación de Bosques de la Zona de Vida Silvestre del PHG.

\section{Marco Teórico}

El Sistema de Clasificación de Ecosistemas del Ecuador Continental (Aguirre, 2012) distingue 62 ecosistemas vegetales para el Ecuador continental, dentro de los cuales se encuentra el BsT. Según Blackie et al. (2014) existe muy escasa literatura disponible sobre el BsT, principalmente por las dificultades que existen para definirlo. Sin embargo, se pueden definir como ecosistemas donde "la mayoría de especies arbóreas pierden el follaje y unas pocas especies permanecen con follaje, en la temporada seca son escasos los arbustos y hierbas" (Aguirre \& Kvist, 2005). En estos ecosistemas también existen períodos de dormancia cambial (Rozendaal, D., 2010) por una prolongada estación seca. Adicionalmente, tienen climas agradables suelo fértil, apto para la agricultura y la ganadería (Carvajal-Vanegas \& Calvo-Alvarado, 2013), y recientemente para proyectos de mega-turismo (Portillo-Quintero \& Sánchez-Azofeifa, 2010). En Ecuador, el BsT forma parte de dos núcleos: los valles interandinos del norte de Perú, Bolivia y Ecuador y costa del Pacífico de Ecuador y Perú.

La región comprendida en la costa del Pacífico de Ecuador y Perú, conocida también como región Pacífico Ecuatorial (Linares-Palomino et al., 2011), ha sido poco estudiada, a pesar de que las especies leñosas están razonablemente bien conocidas en términos florísticos (Espinosa et al., 2012). Adicionalmente, existen remanentes distribuidos de norte a sur en las provincias de Esmeraldas, Manabí, Guayas, Santa Elena y El Oro. Durante 3 o 4 meses el bosque mantiene denso follaje, el mismo que pierde durante 8 o 9 meses de prolongada sequía (Valverde \& Pérez, 2012). Sin embargo, la estructura y composición del BST se encuentra también dependen también del tipo de historia e influencia humana (Muñoz et al., 2014).

Elementos característicos del BST ecuatoriano como la densidad, área basal, altura de dosel, entre otros, dependen en parte Con respecto a su composición, la familia FABACEAE es la dominante, teniendo abundancias muy elevadas y estando representada por un gran número de géneros y especies (Linares-Palomino et al., 2010; Meira, 2011). Valverde \& Pérez (2012), mencionan que en el BsT predominan las especies leguminosas con hojas compuestas para evitar la pérdida de agua por transpiración y especies con tallos abombados y suelos con poca cobertura. Por otro lado, la estructura del BsT está determinada por los factores que influyen sobre el crecimiento de los individuos.

El crecimiento de las especies de bosque seco está gobernado por la disponibilidad de agua que existe en el suelo, que regirán los mecanismos que poseen las especies para resistir la escasez de agua durante la época seca (Chatuverdi et al., 2012). Otro factor que influye en el crecimiento de las especies del BsT es la cantidad de hojas que se encuentran 
disponibles para la fotosíntesis, que depende a su vez de la disponibilidad de luz, humedad del suelo, capacidad de almacenamiento de agua en el tallo, entre otros (Giraldo \& Holbrook, 2011). A pesar que la bibliografía al respecto del ritmo de crecimiento de los bosques secos tropicales es limitada, han existido esfuerzos para determinar los resultados del manejo en áreas protegidas de BsT.

Un estudio llevado a cabo en el Parque Nacional Santa Rosa en Costa Rica determinó diferentes incrementos medios anuales de las especies encontradas, dependiendo de su estadío sucesional. De acuerdo a Carvajal-Vanegas y Calvo-Alvarado (2013) el incremento medio anual en un período de seis años fue de $1,60 \mathrm{~mm} /$ año para el estadío temprano, $2,20 \mathrm{~mm} / \mathrm{año}$ para el intermedio y $1,20 \mathrm{~mm} /$ año para el tardío, con incrementos mayores de acuerdo a la clase diamétrica. Por su parte Muñoz et. al (2014) identificaron en el BsT de la quinta experimental "El Chilco" en el suroccidente del Ecuador, una distribución diamétrica en forma de "J" invertida, característica de bosques en recuperación, como el BsT ecuatoriano que ha sufrido daños por actividades humanas como la agricultura y el pastoreo, invasión de pastos exóticos, incendios forestales, entre otros (Calvo-Alvarado et al., 2013). Adicionalmente, existen otras relaciones diamétricas y de crecimiento que influyen sobre la estructura del BsT.

Con respecto al crecimiento de los árboles, Ghazoul y Sheil (2010) mencionan que árboles emergentes y de dosel tienen por lo general un mayor incremen- to a mayores diámetros. Por otro lado, Iida et al. (2010) destacan que la relación entre el DAP y la altura es una de las mediciones más comunes, y una de las estrategias más usadas para describir especies de árboles. Iida et al. (2010) destacan además que las especies dominantes en un área determinada tienen por lo general una trayectoria lineal en la relación DAP-altura. Por otro lado, las especies suprimidas tienen una trayectoria curva, donde el crecimiento del DAP decrece más que el crecimiento de la altura. Adicionalmente, es posible evidenciar la competencia que existe en el ecosistema a través del crecimiento de los árboles.

De acuerdo a Weng et al. (2015) los árboles utilizan su tallo leñoso para sobrepasar a sus vecinos cuando hay competencia por luz, teniendo entonces especies de crecimiento altitudinal más rápido que su diámetro. Conocer estas relaciones y los resultados obtenidos en otros lugares de manejo de BsT permite evaluar el éxito del manejo realizado en el PHG, que busca reconstruir los ecosistemas de la Antigua Provincia de Guayaquil. Aunque no se conocen sus límites exactos, se considera que estuvo conformada por toda la costa ecuatoriana, con excepción de la provincia de Esmeraldas (Prentice, 2012). Por este motivo incluye ecosistemas de gran importancia ecológica para la región debido a su endemismo y gran diversidad.

Los bosques secos del sur del Ecuador y norte del Perú, están caracterizados por una alta diversidad y un alto endemismo de diferentes taxas (Linares-Palomino et 
al., 2011). En la Región Pacífico-Ecuatorial, el $21 \%$ de las especies reportadas son endémicas, lo que significa, dentro del contexto de los bosques secos tropicales del Neotrópico, un nivel intermedio de endemismo (Linares-Palomino et al., 2011) con 136 endémicas en Ecuador, de 239 identificadas (Espinosa et al., 2012). Sin embargo, a pesar de su alto nivel de diversidad y endemismo, y su importancia ecológica y económica, el BsT no ha sido capaz de salvarse de la degradación causada por actividades humanas.

Debido a su proximidad con zonas urbanas, son ecosistemas preferidos para asentamientos humanos (Neves et al., 2010), convirtiéndolos en "uno de los ecosistemas más deforestados y menos protegidos en América" (Neves et al., 2010; Sánchez-Azofeifa et al., 2011). Por este motivo, y por la falta de investigación realizada en comparación con los bosques húmedos, varias especies están incluidas en la Lista Roja de Especies Amenazadas de la Unión Internacional para la Conservación de la Naturaleza (IUCN, por sus siglas en inglés).

De acuerdo a Linares-Palomino et al. (2010) 33 especies se encuentran catalogadas dentro de alguna categoría en la Lista Roja de la IUCN, con 30 endémicas a Ecuador, Perú o el Pacífico Ecuatorial, $\mathrm{y}$ tres con una distribución geográfica mayor. De esta lista, doce (12) se encuentran en peligro (EN), diez (10) vulnerables (VU), tres (3) casi amenazadas (NT), una (1) preocupación menor, seis (6) con datos insuficientes (DD) y una (1) no evaluada (NE). Por este motivo, la ZVS del PHG tiene una gran importancia ecológica al contribuir con el manejo de especies en alguna categoría de conservación.

Para el manejo del BsT se elaboró un Plan de Manejo Ambiental con las directrices para el manejo de la ZVS. Algunas de éstas incluyen: mejoramiento de suelo, a realizarse en época seca; podas de formación, mantenimiento y fitosanitarias, entre otros. Debido a la importancia económica, social, cultural y ecológica de los bosques secos tropicales para el país, es necesario evaluar el estado actual de las áreas de bosque seco tropical de PHG, para analizar y mejorar el manejo que se ha dado desde el año 2002.

\section{Área de Estudio}

El PHG inaugurado en 1999 está ubicado en el cantón Samborondón, Parroquia Satélite La Puntilla, km 1 1 1/2 y Avenida Esmeraldas, junto a la ciudadela Entre Ríos y al margen izquierdo del río Daule. Cuenta con ocho hectáreas divididas en tres zonas: Zona de Vida Silvestre (ZVS), Zona Urbano Arquitectónica y Zona de Tradiciones (Córdova, 2011). Los parches de bosque seco tropical se encuentran ubicados en la ZVS.

La ZVS tiene una extensión de 3,25 hectáreas, con una infraestructura que consiste en una red de puentes diseñados en función de los diferentes escenarios naturales que se presentan. En este espacio se exhiben flora y fauna representativas de la Antigua Provincia de Guayaquil, como el Bosque de Manglar, Bosque de Llanura Inundable, Bosque de Garúa y Bosque Seco Tropical (Córdova, 2011). 


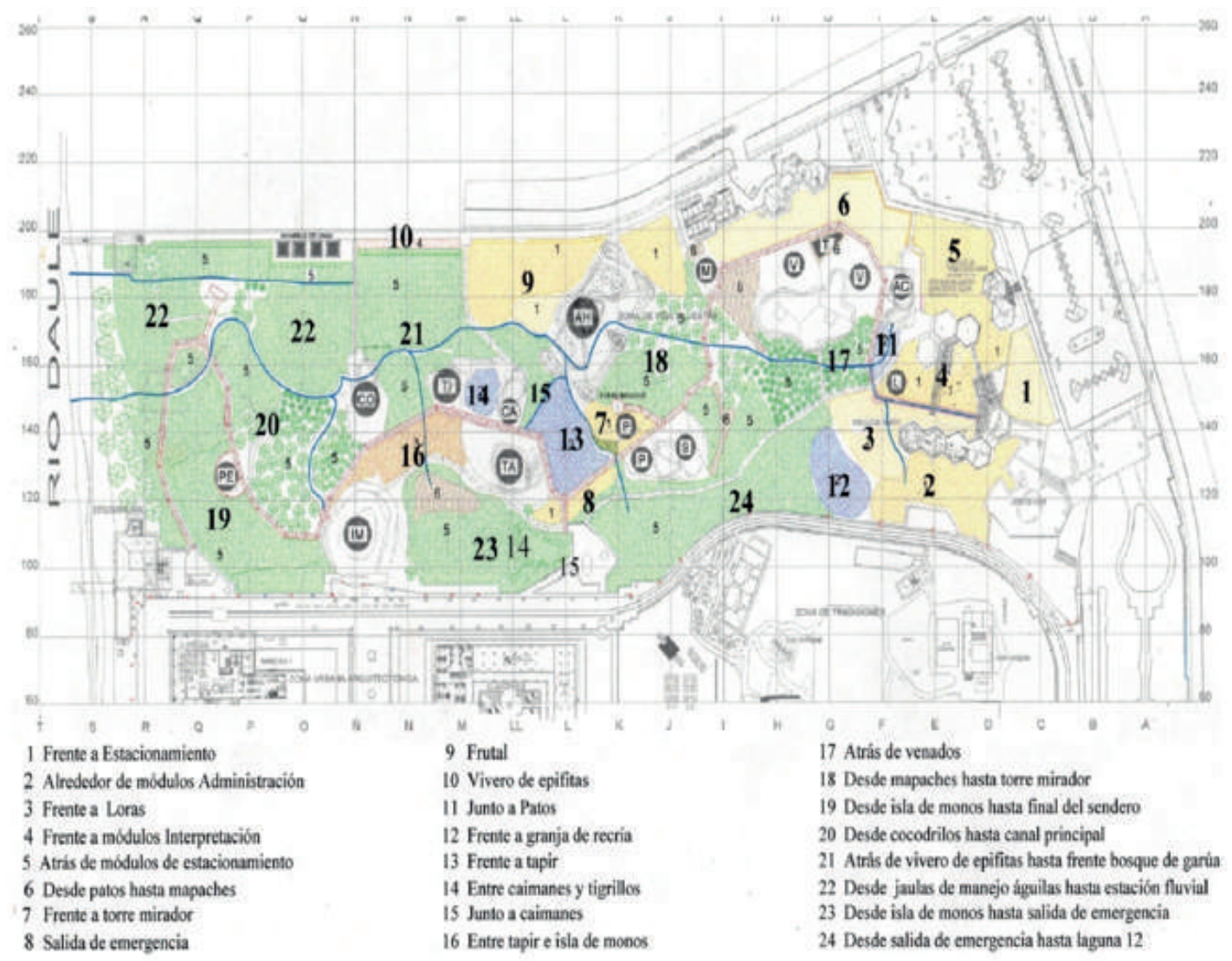

Figura 1. Sectorización de bosques del PHG (Molina \& Molina, 2007).

El bosque seco tropical tiene una extensión de 6,200 $\mathrm{m} 2$, donde había un bosque natural con grandes extensiones dominadas por majagua (Ibiscus tiliacius), así como árboles de fernán sánchez (Triplaris cumingiana), tinto de bajos (Pithecellobium daulense), almendros (Terminalia cattapa), nigüitos (Mutingia calabura); guasmos (Guazuma ulmifolia) y arbustos espinosos de la familia Mimosaceae, entre otros (Molina y Molina, 2005). Adicionalmente, la ubicación del PHG contribuye a las características de los ecosistemas que forman parte de la ZVS, al contar con dos estaciones muy marcadas (Elite Consultor Cia. Ltda., 2013).

\section{Metodología}

Esta investigación fue un estudio mixto, descriptivo. Para actualizar el inventario se tomaron fotografías de la vegetación leñosas, se identificaron y se contaron en sitio cada individuo en los 6,200 $\mathrm{m} 2$ de bosque seco, divididos en cinco zonas establecidas con números del 2 al 6. Con la lista elaborada se comparó el número de especies y de individuos con la lista de Molina y Molina (2005). Para registrar los parámetros estructurales del bosque, se incluyeron aquellos individuos que cumplieron con los siguientes parámetros: especies con un Diámetro a la Altura del Pecho (DAP) 
superior o igual a $2,5 \mathrm{~cm}$ que hayan sido sembradas o germinado espontáneamente a partir del 2002. La información se reco- piló en campo con los datos presentados en la tabla 1.

Tabla 1.

Registro Evaluación del Bosque Seco Tropical del PHG.

\begin{tabular}{|l|l|l|l|l|l|l|}
\hline No. & Especie & Perímetro & Altura & Copa & Sotobosque & Observaciones \\
\hline & & & & & & \\
\hline
\end{tabular}

Para determinar el perímetro de los individuos se utilizó una cinta métrica y se midió la circunferencia a la altura del pecho $1,3 \mathrm{~m}$ sobre el terreno, y siguiendo las metodologías específicas propuestas por la Organización de las Naciones Unidas para la Alimentación y la Agricultura (FAO, por sus siglas en inglés) en el Manual de Campo para un Inventario Forestal Nacional (2004). Los valores de DAP se obtuvieron convirtiendo el perímetro de la circunferencia (CAP) en su diámetro (DAP) con la siguiente fórmula: $\mathrm{DAP}=\mathrm{CAP} / \pi$.

La medición de la altura y la copa por su parte, fue realizada con los instrumentos sugeridos por la FAO (2004). Para determinar la altura se realizaron aproximaciones a partir de la altura del investigador, midiéndose la altura total del árbol, desde la base hasta el ápice. Por su parte la copa fue estimada ubicando al investigador al pie del individuo en el fuste, y observando la apertura de la copa, tomando un brazo como referencia para la estimación.

Para elaborar el catálogo se utilizaron las fotografías tomadas en campo de las especies identificadas a nivel de familia género y especie. Se utilizó el programa Canva para presentar las fotografías, según el orden filogenético de la clasificación del Grupo Filogénetico de Angiospermas III (APG III por sus siglas en inglés).

Por último, para evaluar el cumplimiento del Plan de Manejo Ambiental para la Zona de Vida Silvestre, y proponer nuevos lineamientos, se analizaron los programas y subprogramas, así como los resultados del manejo en la estructura y composición del BsT en el PHG.

\section{Resultados y Discusión}

\section{PHG \\ Inventario del Bosque Seco Tropical del}

Se identificaron un total de 378 individuos $\geq$ 2,5 DAP, distribuidos en 26 familias y 62 especies en las cinco zonas del Bosque Seco Tropical del parque (Tabla 2, Anexo 1). La zona con mayor número de individuos fue la Zona 6 , con 153 individuos, que representan el $40,47 \%$. Por otro lado, la Zona 1 posee un menor número de individuos, con un total de 31 , cerca del $8 \%$ del total. La mayoría de los individuos $(61,90 \%)$ encontrados pertenecen a cinco familias: FABACEAE, BIGNONIACEAE, LECYTIDACEAE, RUBIACEAE $\mathrm{y}$ MELIACEAE (Figura 2). 


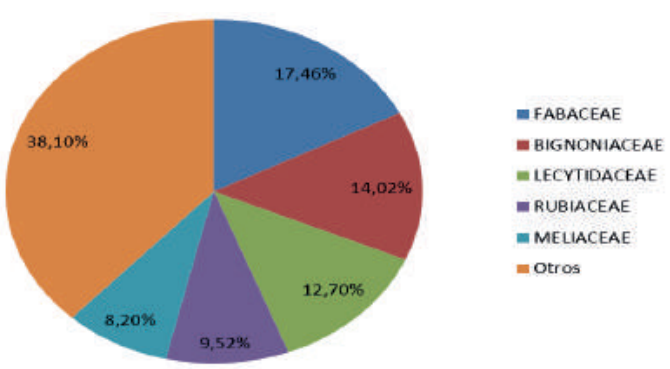

Figura 2. Distribución porcentual de las familias encontradas en el bosque seco tropical del PHG

La familia FABACEAE posee un mayor número de individuos, con 66 individuos, representando el 17,46\%. Los porcentajes y el número de individuos para las otras cuatro familias son: BIGNONIACEAE, 53 individuos y $14,02 \%$; LECYTHICIDAE, 48 individuos y $12,70 \%$; RUBIACEAE, 36 individuos y 9,52\%, y MELIACEAE, que cuenta con 31 individuos que representan el $8,20 \%$ de la muestra. Las otras 21 familias tienen menos del $8 \%$ de individuos en el parque, suman aproximadamente el 37\%. En la Figura 3 se observa el número de individuos por familia, en cada una de las zonas del bosque seco tropical del parque.

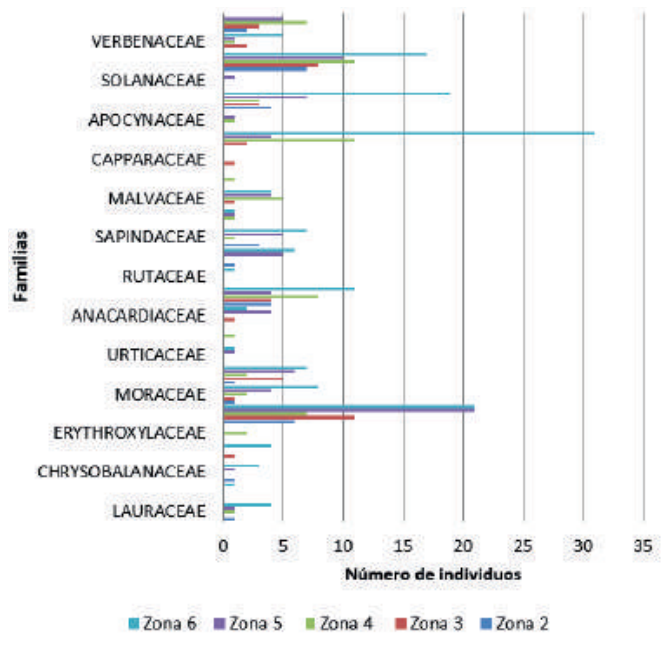

Figura 3. Número de individuos por familia por zona del bosque seco tropical del PHG
La figura anterior muestra cómo se encuentran representadas las 26 familias y 62 especies del BsT en el PHG. La mayoría de individuos pertenecen a la familia FABACEAE, y se encuentran en las Zonas 5 y 6 . Adicionalmente se observa que la familia LECYTHIDACEAE posee el mayor número de individuos por familia y por especie en la zona 6 con 31 individuos de Gustavia angustifolia.

Los resultados promedio por especie de las mediciones dasométricas se encuentran en la Tabla 3 (Anexo 2). Los valores de DAP varían entre 4, 77 a 103,13 distribuidos en siete rangos diamétricos (Figura 4).

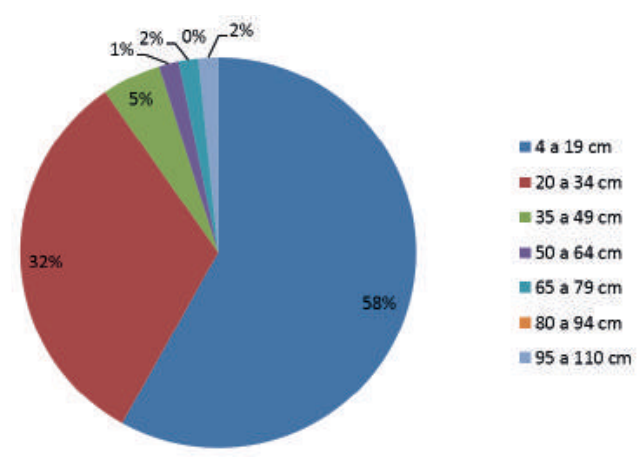

Figura 4. Rango de distribución DAP de la vegetación leñosa bosque seco tropical del PHG

E1 90\% de las especies se encontraron entre los 4 a $34 \mathrm{~cm}: 58 \%$ están en el rango de 4 a $19 \mathrm{~cm}$, y $32 \%$ entre los 20 a $34 \mathrm{~cm}$. Para la altura se establecieron cinco rangos, ya que todas las especies se encuentran entre los 1,50 y 15,00 m. $78 \%$ de los individuos están entre los rangos de 3 a 9 metros: $39 \%$ se encuentra entre 3,00 a $6,00 \mathrm{~m}$, y $39 \%$ de 6,00 a $9,00 \mathrm{~m}$ (Figura 5) y el 53\% de los individuos presentaron una copa entre 2 a 4 m (Figura6). 


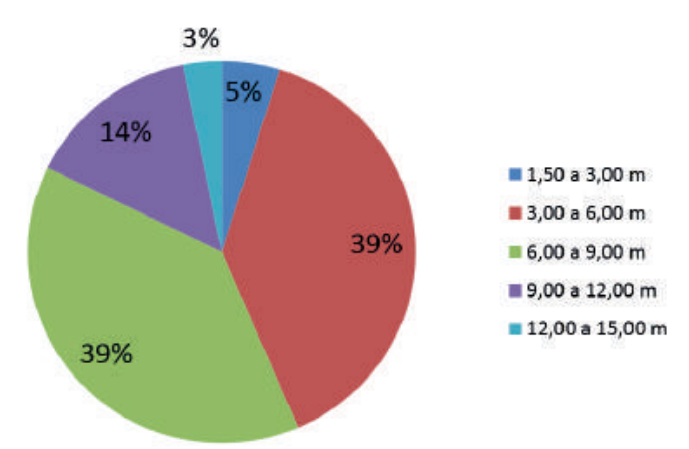

Figura 5. Rango de distribución de altura de la vegetación leñosa del bosque seco tropical del PHG

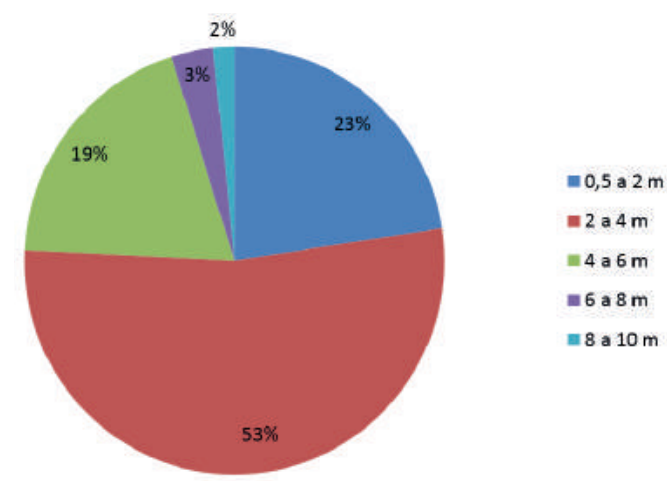

Figura 6. Rango de distribución copas de la vegetación leñosa del bosque seco tropical del PHG

La mayoría de individuos encontrados tienen una copa entre los 2 a $4 \mathrm{~m}(53 \%)$, que junto al rango anterior de 0,5 a $2 \mathrm{~m}(23 \%)$ representan el $76 \%$ de la muestra. Únicamente el $5 \%$ de las especies se encuentran entre los 6 y $8 \mathrm{~m}$ de copa, $3 \%$ entre 6 a $8 \mathrm{~m}$ y el $2 \%$ entre 8 a $10 \mathrm{~m}$.

Debido a la falta de registros e inventarios de la vegetación leñosa en el bosque seco tropical del PHG, no fue posible evaluar tasa de crecimiento de los individuos que fueron sembrados en el proyecto de zonificación del parque. Sin embargo, a partir de estos resultados se podrá continuar el monitoreo del crecimiento de la vegetación leñosa que se ha establecido desde el 2002 hasta el 2015.

Al comparar las listas de la vegetación leñosa del 2005 en la que se registraron 460 individuos, al 2015 se registraron 378 individuos lo que evidenció una pérdida de 82 individuos que corresponde al 18\%. Se desconocen las causas que produjeron esta pérdida, debido a que no se encontraron registros históricos que evidencien desde cuando ocurrió y cuales factores naturales cantropogénicos afectaron esos individuos. Por ejemplo, se observaron tocones de árboles, dentro de encierros de fauna establecidos posteriores a la restauración del bosque.

Entre los factores naturales, la restauración ecológica del bosque seco generó competencia entre las especies y probablemente algunos de los individuos no se pudieron establecerse a pesar de las condiciones favorables, determinadas en el Plan de Manejo del Bosque.

En cuanto a los parámetros estructurales del bosque se evidenció que el DAP promedio de la mayoría de los individuos se encuentran entre los 4 a $19 \mathrm{~cm}$ de DAP, con un total de 36 especies (52\%). Erythroxylum glaucum y Cordia alliodora presentaron los menores valores DAP mientras que Sweetenia macrophyllia. Y Pseudobombax millei presentaron los mayores valores de DAP.

Los rangos diamétricos presentan una curva de jota invertida lo que sugieren que el BsT de la Zona de Vida Silvestre del PHG es un bosque joven en proceso de recuperación con especies de crecimiento lento, en su mayoría especies maderables. 


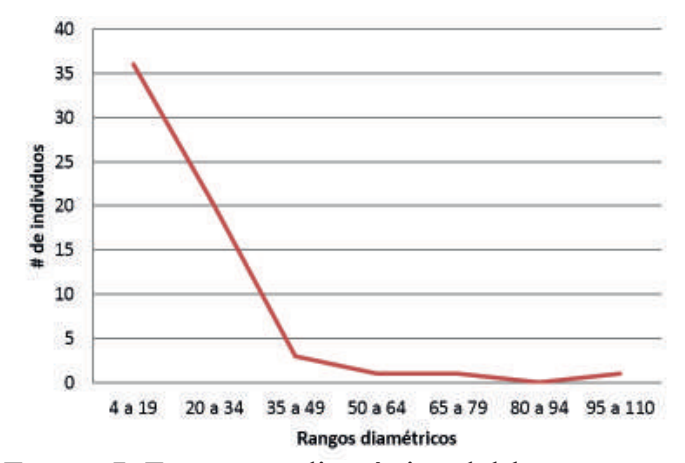

Figura 7. Estructura diamétrica del bosque seco del PHG Sep-Dic 2015

La mayoría de especies (48), se encuentran entre los 3 y 9 metros de altura únicamente Capparidastrum bonifazianum, Cynometra bauhiniifolia y E. glaucum son menores 0 iguales a tres metros. P. millei y Anacardium sp. son las únicas que se encuentran sobre los 12 metros de altura. Esto comprueba el crecimiento rápido del $\mathrm{P}$. millei en condiciones favorables. Estos resultados presentan información adicional sobre el comportamiento de los individuos en este ecosistema.

A pesar que los diámetros de las especies sugieren que es un bosque joven y en crecimiento, también existe una alta competencia por disponibilidad de luz acelerando su crecimiento vertical. Esta puede ser la causa de que los rangos diamétricos presentan una curva en "J" invertida, mientras que la altura no.

A pesar de la falta de datos para evidenciar el crecimiento a través del tiempo, los resultados indican una trayectoria lineal en la relación DAP-altura, característica de especies dominantes en un ecosistema. La falta de una trayectoria lineal en la relación DAP-altura en las demás especies evidencia también que se ven suprimidas y tienden a tener un ritmo de crecimiento del DAP menor al ritmo de crecimiento de su altura.

\section{Plan de Manejo Ambiental}

El Plan de Manejo de Bosques de la Zona de Vida Silvestre del PHG tiene como objetivo "elaborar un programa de manejo integral y sustentable de los componentes de cada ecosistema, que permita su conservación y uso racional" (Molina \& Molina, 2007). Por tal motivo, se desarrolló un Programa de Manejo Ambiental, que contiene el Sub-programa de Mantenimiento del Bosque Seco Tropical. En este Subprograma, cuyo objetivo fue "normar las actividades de mantenimiento para la conservación óptima de los bosques" (Molina \& Molina, 2007), se incluyen las siguientes actividades:

$\begin{array}{ll}\text { - } & \text { Mejoramiento de suelo } \\ \text { - } & \text { Poda } \\ \text { - } & \text { Riego } \\ \text { - } & \text { Rlineamiento } \\ \text { - } & \text { Deshierba y reubicación de plantas } \\ \text { - } & \text { Limpieza de plantas } \\ \text { - } & \text { Control de plagas } \\ \text { - } & \text { Reproducción de plantas } \\ & \text { Siembra y/o trasplante }\end{array}$

A pesar de existir un Plan de Manejo de Bosques para el Bosque Seco Tropical en el PHG, éste no se evidenció su cumplimiento a través de hojas de registro de las actividades o evidencias fotográficas. Durante la fase de campo se evidenció que sí ha existido mejoramiento del suelo, así como actividades constantes de poda, alineamiento, raleo, deshierba y control de plagas. Sin embargo, éstas no cuentan con los medios de verificación necesarios para evidenciar su cumplimiento tal como ha sido establecido en el plan de manejo. 
Además de esto, durante el desarrollo del trabajo de campo se evidenciaron podas y cortes a especies que no tienen registro 0 justificación del corte y el establecimiento de nuevos encierros para exhibición de fauna, donde se perdieron especies sembradas en el año 2002, lo hizo perder la barrera visual de vegetación que existía entre el Parque y su entorno, que evitaba la visualizan de edificaciones como el Teatro Sánchez Aguilar.

A pesar de los problemas de registro histórico de información del bosque y del cumplimiento de las medidas del Plan de Manejo, el BsT ha logrado establecerse con las especies que han sobrevivido. Sin embargo, es necesario realizar una actualización del Plan de Manejo con propuestas adaptadas al estado actual de los ecosistemas para contribuir a su conservación.

Por lo expuesto, se propone actualizar el Plan de Manejo e incluir los siguientes lineamientos:

1. Realizar diagnósticos del entorno para mitigar efectos visuales y auditivos dentro del parque.

2. Establecer monitoreo y seguimiento de la fauna que interactúa con los árboles de la Zona de Vida Silvestre.

3. Elaborar una Planificación Anual con indicadores verificables (e.g. número de podas programadas/ número de podas elaboradas) de manera periódica con base en los lineamientos del Plan de Manejo.

4. Mantener el registro del cumplimiento del Cronograma de Actividades Semanal, elaborado en base a la Planificación Anual.

5. Mantener un inventario forestal anual, con mediciones dasométricas tomadas cada dos años con el fin de poder evaluar el crecimiento de los individuos.
6. Implementar estrategias (e.g. siembras luego de la elaboración del inventario anual) que logren mantener una mejor representatividad de todas las especies.

7. Las estrategias deben ser implementadas en base al número de individuos en todas las zonas de la Zona de Vida Silvestre del PHG, respetando el espacio necesario para cada individuo.

8. Establecer programas de donaciones voluntarias para contribuir al financiamiento de las actividades del parque (e.g. programas de adopción de árboles de la ZVS).

\section{Conclusiones}

El bosque seco se ha establecido con 62 especies y 26 familias de vegetación leñosa, a pesar de que se ha perdido el $18 \%$ de los individuos sembrados en la restauración del bosque desde el año 2002. La familia FABACEAE con 17 especies presentó la mayor diversidad.

Los parámetros estructurales de la vegetación leñosa presentaron valores de DAP que mostraron una curva en forma de "J" invertida, característica de un bosque joven, debido a que el $39 \%$ se encuentra en el rango entre 4 y $19 \mathrm{~cm}$ de DAP.

P. millei fue la especie con mayor DAP altura y copa, por lo que puede ser consideradas como especie emergente que según Ghazoul y Sheil (2010) son las especies que adquieren mayor altura a mayor diámetro.

No se han ejecutado las medidas del Plan de Manejo establecida para el bosque seco con sus respectivos medios de verificación. La falta de recopilación de información es el mayor limitante. Tampoco se había contempla- 
do una medida que especificara la realización de inventarios anuales o bianuales.

Por este motivo, y para fortalecer el éxito de este proyecto, es indispensable realizar una actualización del Plan de Manejo de Bosques donde se destaque la importancia del monitoreo del crecimiento del bosque para que sea posible su evaluación a largo plazo y así contribuir a la memoria histórica de la Antigua Provincia del Guayas. De esta forma el Parque Histórico no solo cumple con el aspecto social sino también con el objetivo medioambiental de reconstruir bosques encontrados en la región Costa del Ecuador, el verdadero éxito de este proyecto.

\section{Recomendaciones}

- Elaborar y difundir una Guía de Árboles del PHG, donde se incluya información sobre el inventario actual y las especies que se encuentran en la Zona de Vida Silvestre.

- Implementar adecuadamente el Plan de Manejo de Bosques, Programa de Manejo Ambiental, con los medios de verificación que han sido establecidos

- Plantar árboles que sirvan como barrera de ruido y contaminación visual en las áreas que limiten con el área urbana (e.g. Zona 5 que limita con el parqueadero del parque).

- Identificar adecuadamente aquellos individuos que fueron sembrados como parte del proyecto de Zonificación del Bosque de la Zona Silvestre del Parque Histórico y aquellos que han nacido de manera espontánea por incorporación de tierra de sembrado.

- Vincular a la comunidad con el manejo y conservación del bosque seco tropical, a través de medios electrónicos, como publicaciones en redes sociales, y programas de carácter educativo que involucren a la comunidad en el cuidado y preservación del Bosque Seco Tropical ecuatoriano.

- Evaluar la diversidad de especies que existe en cada zona del parque con el fin de repoblar en las zonas de menor diversidad, para incrementar la representatividad de especies en el bosque, cuidando el espacio necesario para cada árbol.

- Elaborar estudios de interacción de fauna silvestre asociada a la ZVS del PHG, para conocer relaciones asociadas al desarrollo del bosque como la floración, fructificación y anidación.

- Implementar en el mediano plazo el estrato herbáceo con especies nativas del BsT.

\section{Referencias}

Aguirre, Z., Betancourt, Y., Geada-López, G., y Jasen-Gonzáles, H. (2013). Composición florística y estructura de los bosques secos y su gestión para el desarrollo de la provincia de Loja. Avances, 15, 2, 134-146.

Aguirre, Z. (2012). Especies forestales: bosques secos Ecuador. MAE/FAO: Finlandia, Quito-Ecuador.

Aguirre, Z. y Kvits, P. (2005). Vegetación de los bosques secos de Cerro Negro-Cazaderos, Occidente de la Provincia de Loja. En: M.A. Vásquez, J.F. Freile y L. Suárez (Eds.). Biodiversidad en los bosques secos de la zona de Cerro Negro-Cazaderos, occidente de la provincia de Loja: un reporte de las evaluaciones ecológicas y socioeconómicas rápidas. Pp. 9-24. EcoCiencia, MAE y Proyecto Bosque Seco: Quito, Ecuador. 
Blackie et al. (2014). Tropical dry forests: The state of global of global knowledge and recommendations for future research. Center for International Forestry Research: Bogor, Indonesia.

Carvajal-Vanegas, D. y Calvo-Alvarado, J. (2013). Tree diameter growth of three successional stages of tropical dry forests, Santa Rosa National Park, Costa Rica. En A. Sanchez-Azofeifa, J. Powers, M. Quesada (Eds.), Tropical Dry Forests in the Americas: Ecology, Conservation and Management (351-365). Boca Raton, Florida: CRC Press.

Chaturvedi, R., Raghubanshi, A. y Singh, J. (2012). Growth of tree seedlings in a tropical dry forest in relation to soil moisture and leaf traits. Journal of Plant Ecology, 1-13, doi: 10.1093/jpe/rts025

Córdova, J. (2011). Estructuración de un manual de procedimientos para la atención al público del PHG. (Tesis inédita de pregrado). Escuela Superior Politécnica del Litoral, Guayaquil, Ecuador.

Espinosa, C., de la Cruz, M., Luzuriaga, A., y Escudero, A. (2012). Bosques tropicales secos de la región Pacífico Ecuatorial: diversidad, estructura, funcionamiento e implicaciones para la conservación. Ecosistemas, 21, 1, 161-179.

Espinoza, E. (2012). Estudio de Impacto Ambiental Expost para el Teatro Sánchez-Aguilar, Recuperado de Gobierno Provincial del Guayas: http://www.guayas.gob.ec/dmdocuments/me- d i 0 - a m b i ente/eia/2012/2012-julio/ESTUDIO $\%$ 20DE\%20IMPACTO\%20AMBIENTA L $\% 20$ TEATRO $\% 20 \mathrm{~S} \% \mathrm{C} 3 \% 81 \mathrm{NCHE}$ Z\%20AGUILAR.pdf

Ghazoul, J. y Sheil, D. (2010). Tropical rain forest ecology, diversity, and conservation. Oxford: University Press.

Giraldo, J. y Holbrook, N. (2011).

Physiological mechanisms underlying the seasonality of leaf senescence and renewal in seasonally dry tropical forests trees En Dirzo, R., Mooney, H., Ceballos, G. y Young, H. (eds.). Seasonally Dry Tropical Forests: Ecology and Conservation, 129-140. Washington, DC: Island Press.

Iida, Y., Kohyama, T., Kubo, T., Kassim, A., Pooter, L., Sterck, F y Potts, M. (2011). Tree architecture and life-history strategies across 200 co-occurring tropical tree species. Functional Ecology, 25, 1260-1268

Jaramillo, V., Martínez-Yrízar, A., Sanford, R. (2011). Primary Productivity and Biogeochemistry of Seasonally Dry Tropical Forests. En Dirzo, R., Mooney, H., Ceballos, G. y Young, H. (eds.). Seasonally Dry Tropical Forests: Ecology and Conservation, 109-128. Washington, DC: Island Press.

Linares-Palomino, Oliveira-Filho, A., Pennington, R. T. (2011). Neotropical Seasonally Dry Forests: Diversity, Endemism and Biogeography of Woody Plants. En Dirzo, R., Mooney, 
H., Ceballos, G. y Young, H. (eds.). Seasonally Dry Tropical Forests: Ecology and Conservation, 3-21. Washington, DC: Island Press.

Linares-Palomino, R., Kvist, L., Aguirre-Mendoza, Z. y Gonzales-Inca, C. (2010). Diversity and endemism of woody plant species in the Equatorial Pacific seasonally dry forests. Biodiversity and Conservation, 19, 169-185. doi: 10.1007/s10531-009-9713-4

Meira, D., Oliveira, F., Vieira, F., Soares, G., Duque, R., D Ângelo, S. y Ferreira, R. (2011) Structural Aspects and Floristic Similarity Among Tropical Dry Forest Fragments with Different Management Histories, Revista Árvore, 35, 131-142.

Mendoza, X. y Molina, N. (2015). Evaluación de la estructura poblacional arbórea del manglar en el Parque Histórico Guayaquil. Investigatio Research Review, 6, 101-120.

Ministerio de Ambiente del Ecuador (2012). Sistema de clasificación de ecosistemas del Ecuador continental. Quito-Ecuador: MAE.

Molina, R., y Molina, N. (2007). Plan de Manejo de Bosquez Zona de Vida Silvestre - Parque Histórico Guayaquil, Guayaquil: Fundación Ecológica Rescate Jambelí.

Molina, R., y Molina, N. (2005). Proyecto zonificación del bosque de la zona de vida silvestre del Parque Histórico Guayaquil del Banco Central del Ecuador. Guayaquil: Fundación
Ecológica Rescate Jambelí.

Muñoz, J., Erazo, S. y Armijos. D. (2014). Composición florística y estructura del bosque seco de la quinta experimental "El Chilco" en el suroccidente del Ecuador, Revista Cedamaz, 4, 1, 53-61.

Neves, F., Braga, R., Espírito-Santo, M., Delabie, J., Fernandes, W., Sánchez-Azofeifa, A. (2010). Diversity of Arboreal Ants In a Brazilian Tropical Dry Forest: Effects Of Seasonality and Successional Stage. Sociobology, $56,1,1-18$.

Parque Histórico Guayaquil. (s.f.). ¿Quiénes somos?. Obtenido de Parque Histórico Guayaquil: http://www.parquehistoric o . g o b. e c/ w e b/i n d e x .php/2014-01-23-15-46-58/quienes-so mos-menu.

Portillo-Quintero, C. y Sánchez-Azofeifa, G. (2010). Extent and conservation of tropical dry forests in the Americas. Biological Conservation, 143, 1, 144-155.

Prentice, M. (2012). Propuesta de investigación para el desarrollo de una exposición de arte itinerante, que rescate la historia de los astilleros, e incremente la afluencia de turistas nacionales y extranjeros en la ciudad de Guayaquil. (Tesis inédita de pregrado). Escuela Superior Politécnica del Litoral, Guayaquil, Ecuador.

Rozendaal, D. (2010). Looking backwards: using tree rings to evaluate long-term growth patterns of Bolivian forest tree. 
Riberalta, Bolivia: PROMAB.

Sánchez-Azofeifa, A. y Portillo-Quintero, C.

(2011). Extent and Drivers of Change of Neotropical Seasonally Dry Tropical Forests. En Dirzo, R., Mooney, H., Ceballos, G. y Young, H. (eds.). Seasonally Dry Tropical Forests: Ecology and Conservation, 45-57. Washington, DC: Island Press.

Valverde, F. y Pérez, J. (2012) La biodiversidad vegetal, como capital natural de la sostenibilidad en la costa ecuatoriana. Guayaquil, Ecuador: Programa Editorial de la M. I. Municipalidad de Santiago de Guayaquil.

Weng, E., Malyshev, S., Lichstein, J., Farrior, C., Dybzinski, R., Zhang, T., Shevliakova, y Pacala, S. (2015). Scaling from individual trees to forests in an Earth system modeling framework using a mathematically tractable model of height-structured competition. Biogeosciences, 12, 2655-2694.

Anexo 1

Tabla 2.

Clasificación botánica de árboles del Bosque Seco del PHG por Zona - Año 2015.

\begin{tabular}{|c|c|c|c|c|c|c|c|}
\hline Familia & Especie & Nombre Común & $\begin{array}{l}\text { Zona } \\
2\end{array}$ & $\begin{array}{l}\text { Zona } \\
3\end{array}$ & $\begin{array}{l}\text { Zona } \\
4\end{array}$ & $\begin{array}{l}\text { Zona } \\
5\end{array}$ & $\begin{array}{l}\text { Zona } \\
6\end{array}$ \\
\hline \multirow{3}{*}{ LAURACEAE } & Nectandra laevis Mez. & Jigua & 0 & 0 & 0 & 0 & 3 \\
\hline & Licaria limbosa (R\&P) Kosterm & Canelo & 1 & 0 & 0 & 0 & 1 \\
\hline & Ocotea sp. & Ocotea & 0 & 0 & 1 & 1 & 0 \\
\hline EUPHORBIACEAE & $\begin{array}{l}\text { Phyllanthus juglandifolius (L) } \\
\text { Wild }\end{array}$ & Culo pesado & 0 & 0 & 0 & 0 & 1 \\
\hline CHRYSOBALANACEAE & $\begin{array}{l}\text { Couepia subcordata Benth. Ex. } \\
\text { Hook F. }\end{array}$ & Guaiji & 1 & 0 & 0 & 1 & 3 \\
\hline HYPERICACEAE & Vismia obtusa Spruce & $\begin{array}{l}\text { Sangre de } \\
\text { gallina }\end{array}$ & 0 & 1 & 0 & 0 & 4 \\
\hline ERYTHROXYLACEAE & $\begin{array}{l}\text { Erythroxylum glaucum O. E. } \\
\text { Schulz }\end{array}$ & Coquito & 0 & 0 & 2 & 0 & 0 \\
\hline & Bauhinia haughtii Wunderlin & Pata de vaca & 0 & 0 & 0 & 2 & 2 \\
\hline & Caesalpinia glabrata Kunth & Cascol & 0 & 1 & 3 & 6 & 4 \\
\hline & Brownea ariza Benth. & Árbol de la cruz & 0 & 0 & 1 & 0 & 0 \\
\hline & Cynometra bauhiniifolia Benth. & Cocobolo & 0 & 0 & 0 & 4 & 1 \\
\hline
\end{tabular}




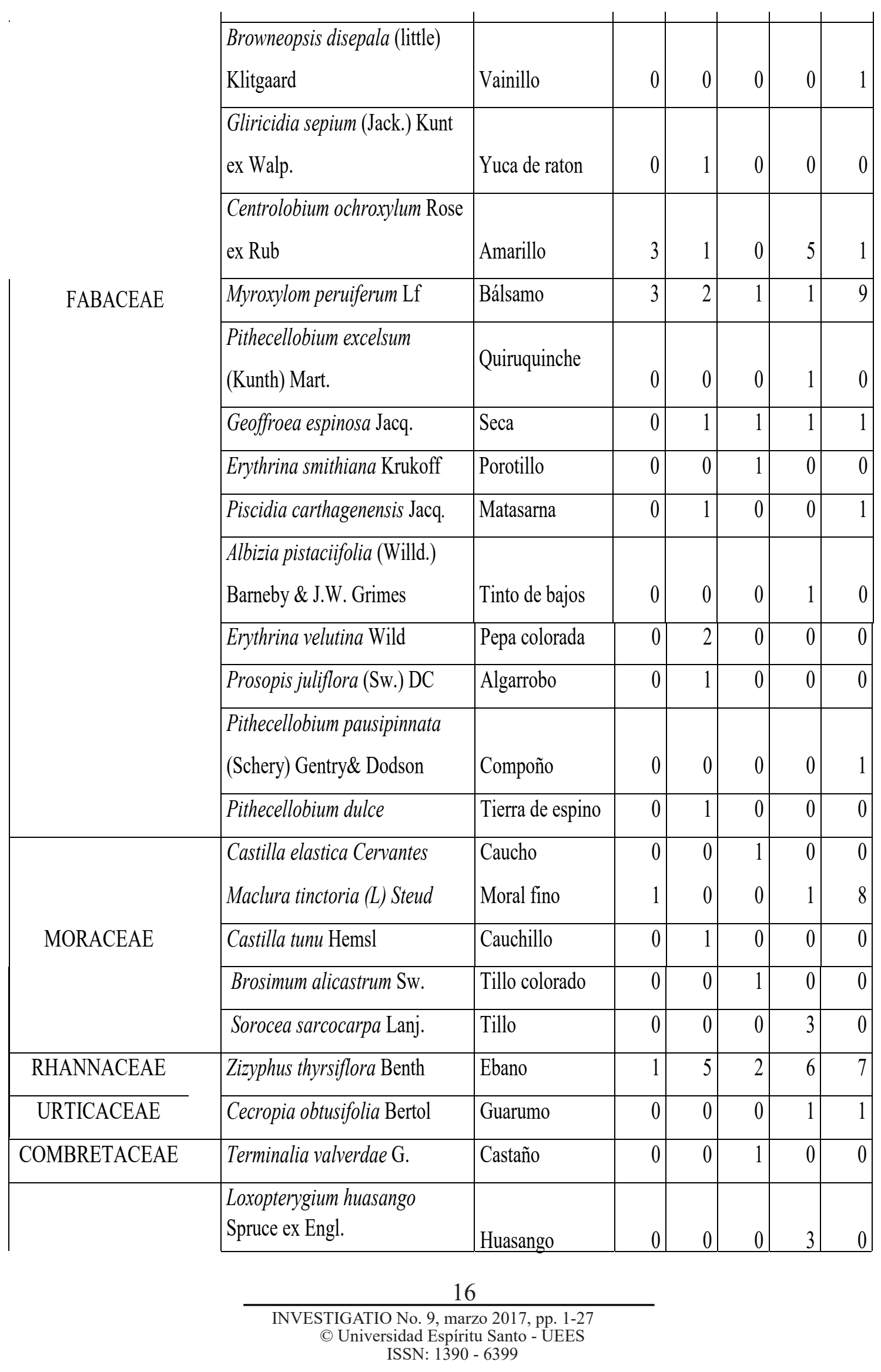




\begin{tabular}{|c|c|c|c|c|c|c|c|}
\hline \multirow[t]{2}{*}{ ANACARDIACEAE } & Spondia mombim $L$. & Ciruelo jobo & 0 & 0 & 0 & 0 & 2 \\
\hline & Anacardium $s p$ & $\begin{array}{l}\text { Marañón } \\
\text { silvestre }\end{array}$ & 0 & 1 & 0 & 1 & 0 \\
\hline \multirow{2}{*}{ MELIACEAE } & Cedrela odorata L. & Cedro & 2 & 3 & 8 & 2 & 8 \\
\hline & Swetenia macrophyllia King & Caoba & 2 & 1 & 0 & 2 & 3 \\
\hline RUTACEAE & $\begin{array}{l}\text { Zanthoxylum setulosum P. } \\
\text { Wilson }\end{array}$ & Sasafras & 0 & 0 & 0 & 0 & 1 \\
\hline BURSERACEAE & Bursera graveolens H.B.K & Palo santo & 1 & 0 & 0 & 5 & 6 \\
\hline SAPINDACEAE & Sapindus saponaria L. & Jaboncillo & 3 & 0 & 1 & 5 & 7 \\
\hline BIXACEAE & $\begin{array}{l}\text { Cochlospermun vitifolium } \\
\text { (Willd.) Spreng. }\end{array}$ & Bototillo & 0 & 0 & 1 & 1 & 1 \\
\hline \multirow{2}{*}{ MALVACEAE } & $\begin{array}{l}\text { Ceiba trichistandra (A. Gray) } \\
\text { Bakh. }\end{array}$ & Ceibo & 0 & 0 & 1 & 1 & 1 \\
\hline & $\begin{array}{l}\text { Cavanillesia platanifolia } \\
\text { (Bonpl.) Kunth }\end{array}$ & Pigio & 0 & 1 & 1 & 0 & 1 \\
\hline
\end{tabular}

Anexo 2

Tabla 3.

Mediciones dasométricas de las especies de Bosque Seco Tropical del PHG

\begin{tabular}{|c|c|c|c|c|c|}
\hline Familia & Especie & $\begin{array}{l}\text { CAP } \\
(\mathrm{cm})\end{array}$ & $\begin{array}{l}\text { DAP } \\
(\mathrm{cm})\end{array}$ & $\begin{array}{c}\text { Altura } \\
(\mathrm{m})\end{array}$ & $\begin{array}{c}\text { Copa } \\
\text { (m) }\end{array}$ \\
\hline \multirow{3}{*}{ LAURACEAE } & Nectandra laevis Mez. & 13,3 & 8,49 & 6,00 & 1,83 \\
\hline & Licaria limbosa (R\&P) Kosterm & 17,8 & 11,30 & 7,50 & 2,00 \\
\hline & Ocotea sp. & 16,8 & 10,66 & 7,00 & 3,50 \\
\hline EUPHORBIACEAE & Phyllanthus juglandifolius (L) Wild & 34,0 & 21,65 & 12,00 & 3,00 \\
\hline CHRYSOBALANACEAE & $\begin{array}{l}\text { Couepia subcordata Benth. Ex. } \\
\text { Hook F. }\end{array}$ & 32,0 & 20,37 & 6,13 & 8,25 \\
\hline ERYTHROXYLACEAE & Erythroxylum glaucum O. E. Schulz & 7,5 & 4,77 & 1,75 & 1,25 \\
\hline & Bauhinia haughtii Wunderlin & 20,3 & 12,89 & 5,75 & 2,75 \\
\hline
\end{tabular}




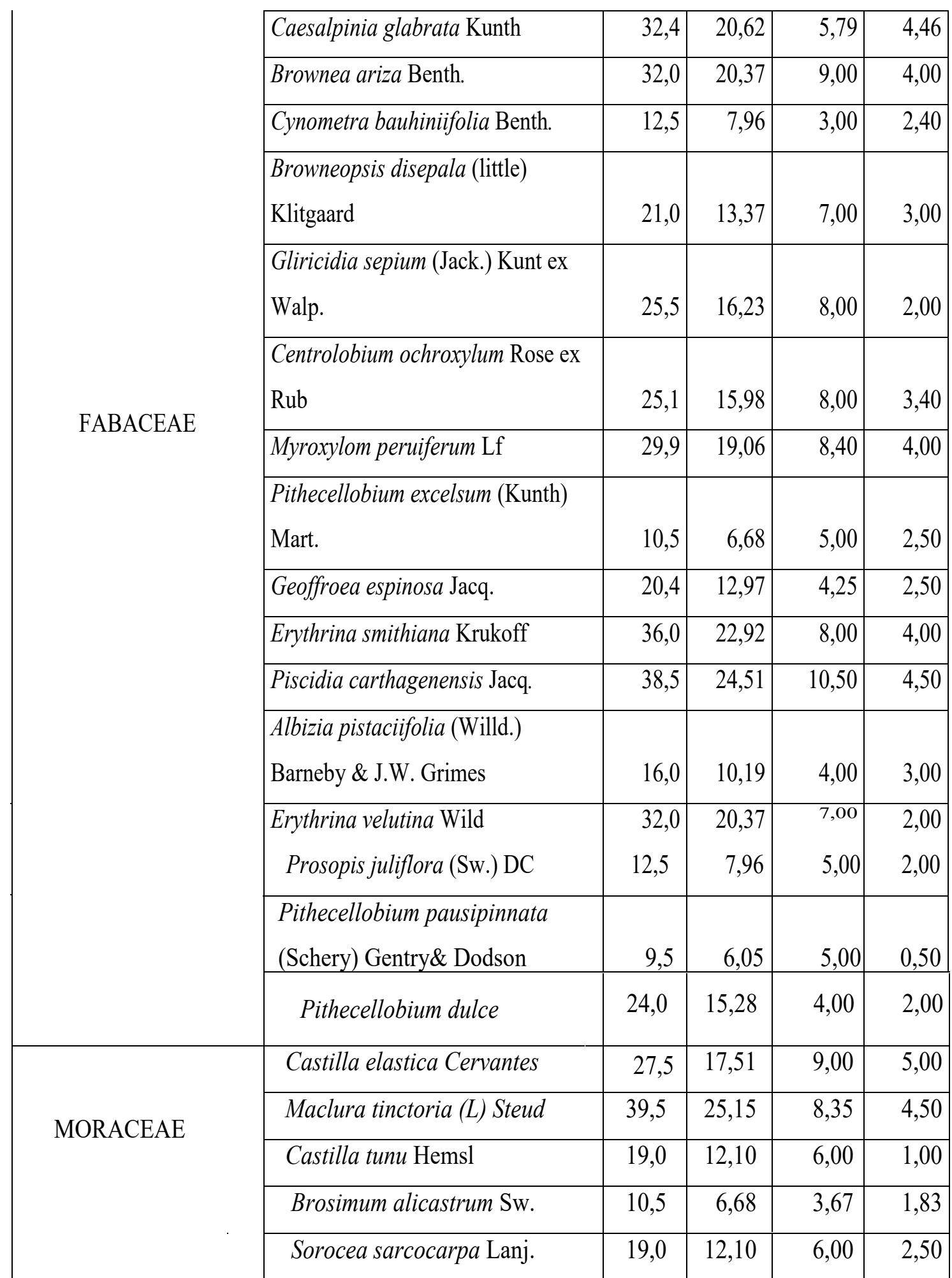




\begin{tabular}{|c|c|c|c|c|c|}
\hline RHANNACEAE & Zizyphus thyrsiflora Benth & 37,3 & 23,74 & 6,98 & 3,98 \\
\hline URTICACEAE & Cecropia obtusifolia Bertol & 39,3 & 24,99 & 10,00 & 1,75 \\
\hline COMBRETACEAE & Terminalia valverdae $\mathrm{G}$. & 50,0 & 31,83 & 12,00 & 6,00 \\
\hline \multirow{3}{*}{ ANACARDIACEAE } & $\begin{array}{l}\text { Loxopterygium huasango Spruce ex } \\
\text { Engl. }\end{array}$ & 32,7 & 20,80 & 7,17 & 3,75 \\
\hline & Spondia mombim L. & 32,5 & 20,69 & 8,00 & 4,50 \\
\hline & Anacardium sp. L. & 42,0 & 26,74 & 15,00 & 6,00 \\
\hline \multirow{2}{*}{ MELIACEAE } & Cedrela odorata $\mathrm{L}$. & 41,1 & 26,14 & 9,24 & 3,98 \\
\hline & Swetenia macrophyllia King & 62,6 & 39,83 & 11,50 & 4,63 \\
\hline RUTACEAE & Zanthoxylum setulosum P. Wilson & 26,0 & 16,55 & 7,00 & 4,00 \\
\hline BURSERACEAE & Bursera graveolens H.B.K & 27,3 & 17,40 & 4,67 & 2,38 \\
\hline SAPINDACEAE & Sapindus saponaria L. & 18,1 & 11,52 & 4,69 & 2,53 \\
\hline BIXACEAE & $\begin{array}{l}\text { Cochlospermun vitifolium (Willd.) } \\
\text { Spreng. }\end{array}$ & 80,8 & 51,46 & 9,00 & 2,67 \\
\hline \multirow{5}{*}{ MALVACEAE } & $\begin{array}{l}\text { Ceiba trichistandra (A. Gray) } \\
\text { Bakh. }\end{array}$ & 47,2 & 30,03 & 5,67 & 2,50 \\
\hline & $\begin{array}{l}\text { Cavanillesia platanifolia (Bonpl.) } \\
\text { Kunth }\end{array}$ & 27,3 & 17,40 & 6,17 & 2,33 \\
\hline & $\begin{array}{l}\text { Pseudobombax millei (Standl) A. } \\
\text { Robyns }\end{array}$ & 162,0 & 103,13 & 15,00 & 7,00 \\
\hline & Hibiscus tiliaceus L. & 33,5 & 21,33 & 6,00 & 3,75 \\
\hline & Guazuma ulmifolia Lam. & 42,8 & 27,25 & 8,00 & 4,80 \\
\hline CARICACEAE & $\begin{array}{l}\text { Vasconcellea parviflora (A. Cd.) } \\
\text { Solms }\end{array}$ & 20,0 & 12,73 & 5,00 & 2,00 \\
\hline CAPPARACEAE & $\begin{array}{l}\text { Capparidastrum bonifazianum } \\
\text { (Cornejo \& Iltis) }\end{array}$ & 13,0 & 8,28 & 3,00 & 2,00 \\
\hline LECYTIDACEAE & Gustavia angustifolia Benth & 14,2 & 9,01 & 4,21 & 1,74 \\
\hline APOCYNACEAE & Vallesia glabra (Cav) Link & 30,0 & 19,10 & 3,25 & 2,50 \\
\hline
\end{tabular}


Juan Carlos Cisneros Bohórquez, Natalia Molina Moreira

\begin{tabular}{|c|c|c|c|c|c|}
\hline \multirow{3}{*}{ RUBIACEAE } & Simira ecuadoriensis (Standl) & & & & \\
\hline & Steger & 18,1 & 11,50 & 5,59 & 2,50 \\
\hline & Alseis eggersi Stadl & 22,0 & 14,01 & 7,00 & 3,00 \\
\hline SOLANACEAE & Acnistus arborescens (L.) Schlecht & 26,0 & 16,55 & 5,00 & 3,00 \\
\hline \multirow{6}{*}{ BIGNONIACEAE } & $\begin{array}{l}\text { Handroanthus chrysanthus } \\
\text { (Jacq.) S.O.Grose }\end{array}$ & 19,5 & 12,44 & 5,57 & 3,38 \\
\hline & $\begin{array}{l}\text { Handroanthus chrysanthus ssp. } \\
\text { pluvicola (A.H. Gentry) S.O. Grose }\end{array}$ & 39,5 & 25,15 & 7,60 & 4,00 \\
\hline & Tabebuia heterophylla (DC.) & & & & \\
\hline & Britton & 21,5 & 13,69 & 4,75 & 2,75 \\
\hline & Tabebuia rosea (Bertol.) DC. & 104,0 & 66,21 & 11,00 & 5,00 \\
\hline & $\begin{array}{l}\text { Handroanthus billbergii (Bureau \& } \\
\text { K.Schum.) S.O.Grose }\end{array}$ & 26,8 & 17,03 & 8,00 & 3,50 \\
\hline VERBENACEAE & Vitex gigantea Kunth & 56,1 & 35,72 & 9,11 & 4,78 \\
\hline \multirow{3}{*}{ BORRAGINACEAE } & Cordia lutea Lam. & 17,2 & 10,94 & 4,00 & 2,50 \\
\hline & $\begin{array}{l}\text { Cordia alliodora (R\&P) Oken } \\
\text { Allgen }\end{array}$ & 30,2 & 19,23 & 7,20 & 3,80 \\
\hline & Cordia macrantha Chodat & 38,0 & 24,19 & 9,00 & 5,00 \\
\hline
\end{tabular}


Anexo 3.

Catálogo Bosque Seco Tropical Zona de Vida Silvestre PHG

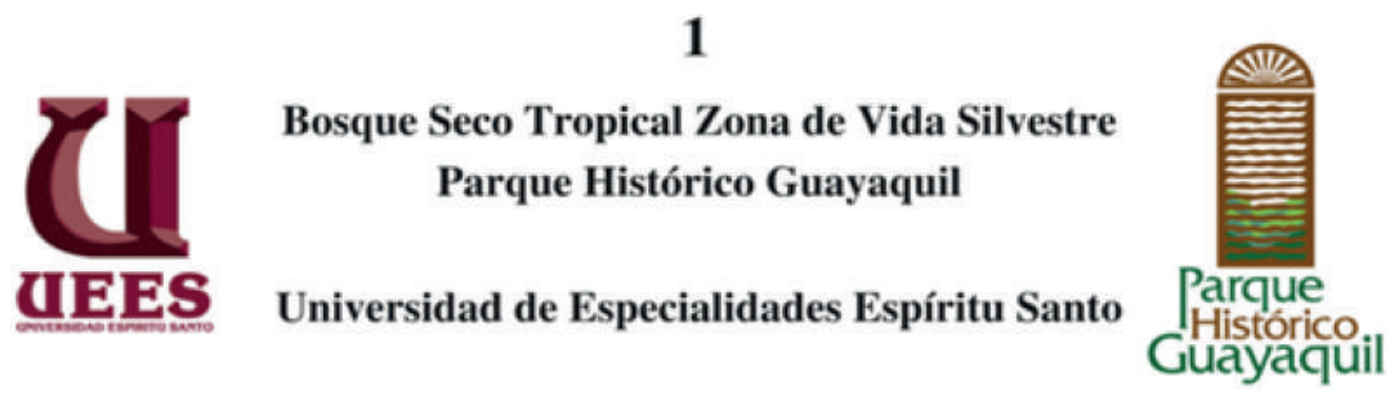

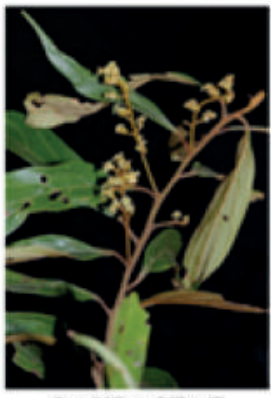

LAURACEAE

Nectandra laevis

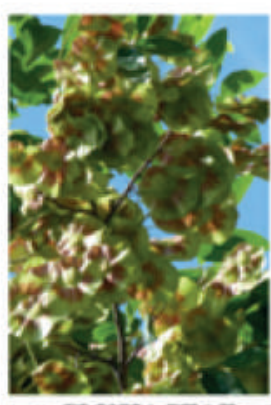

Clusiaceae

Vismia obrusa

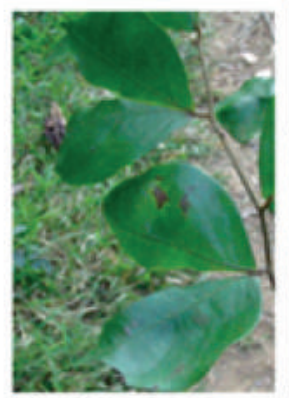

FABACEAE

Cynometra bauhiniüfolia

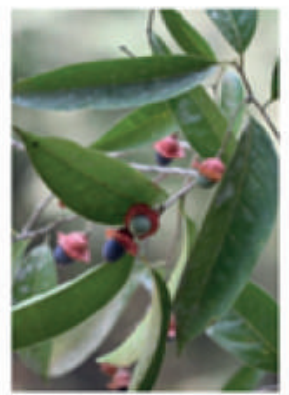

LAURACEAE

Licaria limbosa

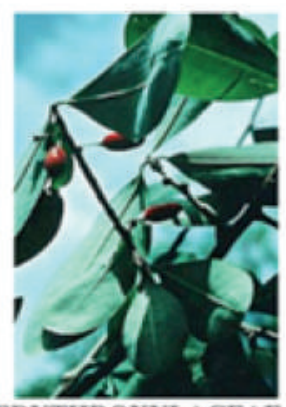

Enthrexwlum glaucum

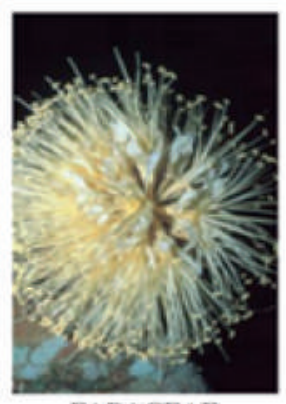

FABACEAE

Browneopsis disepala

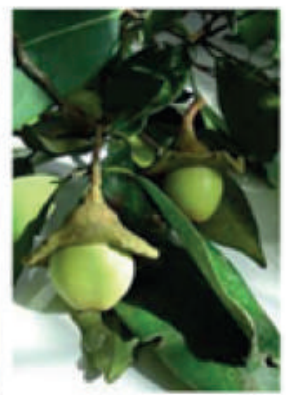

LAURACEAE

Ocotea sp.

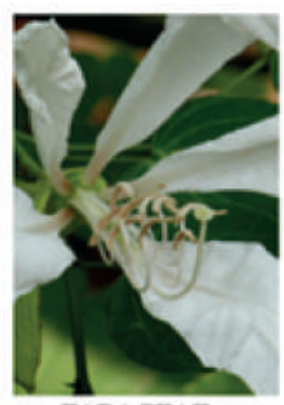

FABACEAE

Bauhinia haughtii

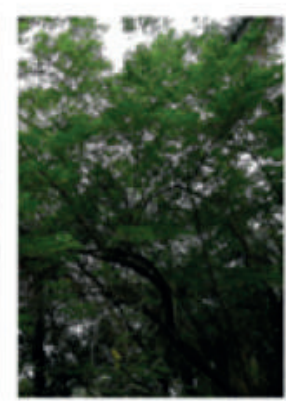

FABACEAE

Gliricidia sepium

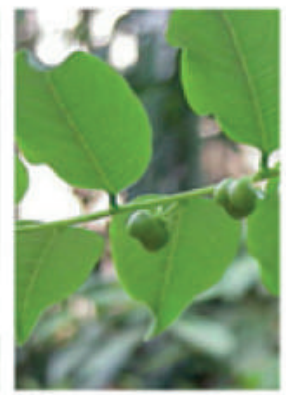

EUPHORBIACEAE CHETSOBAINACEAE Phyllamthus juglandifolius

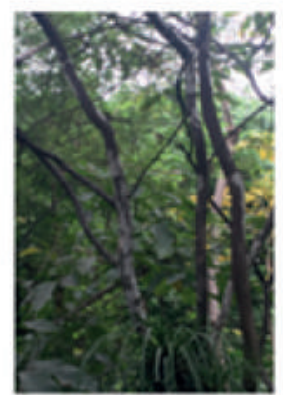

FABACEAE

Caesalpinia glabrata

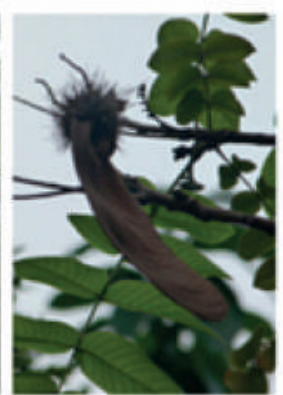

FABACEAE

Centrolobium ochroxylum
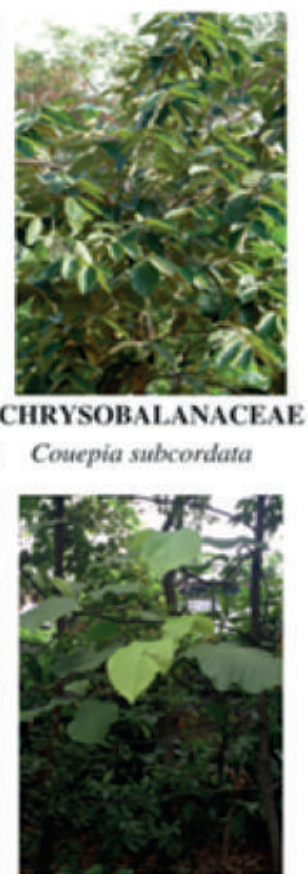

FABACEAE

Brownea ariza

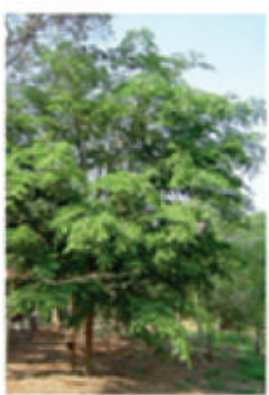

FABACEAE

Myrorylom peruifenum

\section{1}

INVESTIGATIO No. 9, marzo 2017, pp. 1-27

(C) Universidad Espíritu Santo - UEES

ISSN: $1390-6399$ 


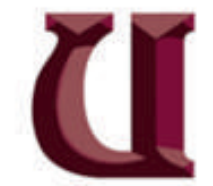

\section{IEES}

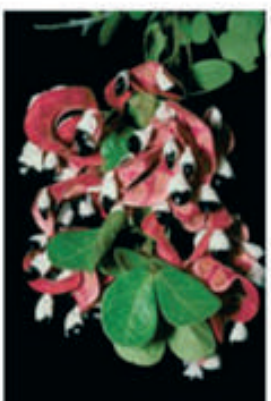

FABACEAE

Pithecellobium excelsum

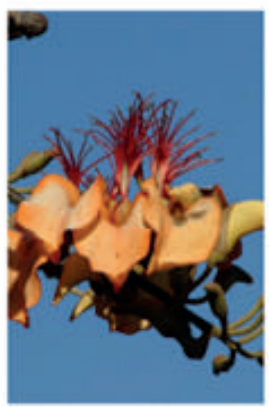

FABACEAE

Erythrina velutina

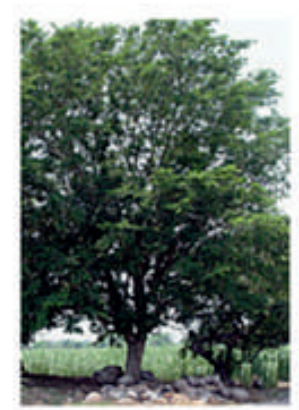

MORACEAE

Maclura tinctoria

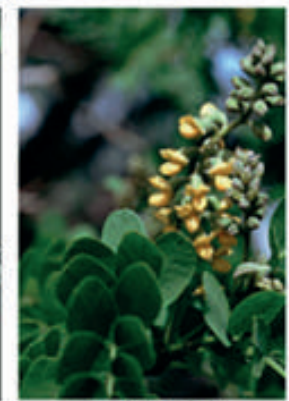

FABACEAE

Geoffroca espinosa

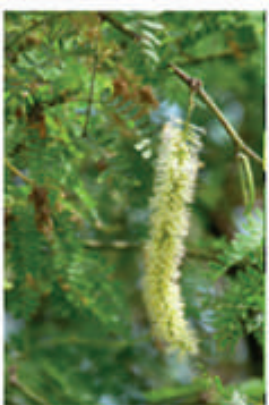

FABACEAE

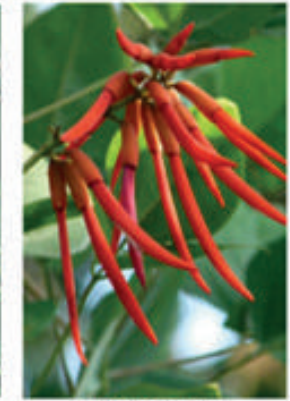

FABACEAE

Enthrina smithiana

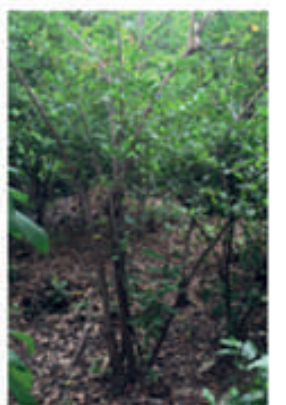

Pithecellobium pausipinnata Pithecellobium dulce

Piscidia carthagenensis

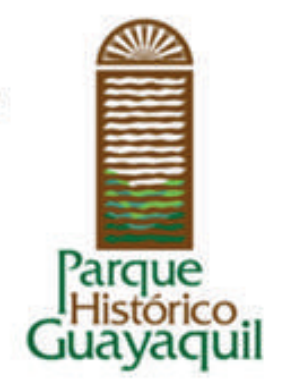

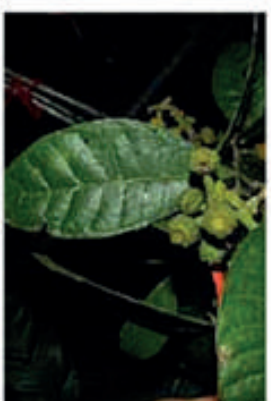

MORACEAE

Castilla tunu

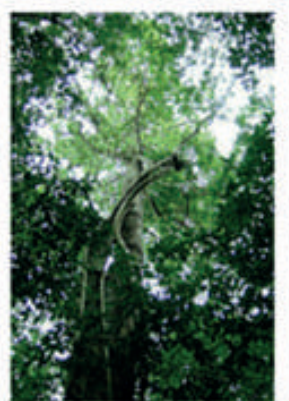

MORACEAE

Brosimum alicastrum

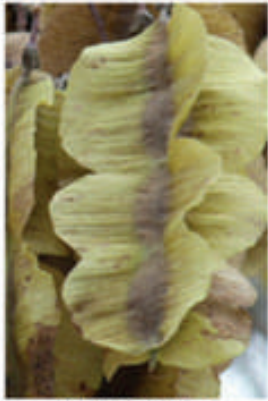

FABACEAE

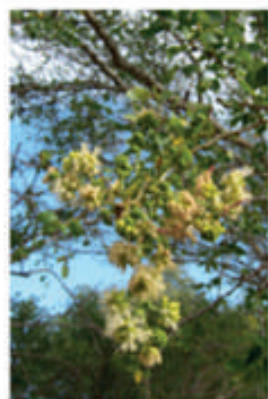

FABACEAE

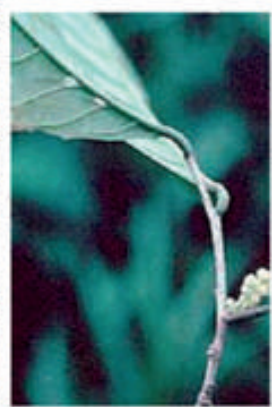

MORACEAE

Sorocea sarcocarpa

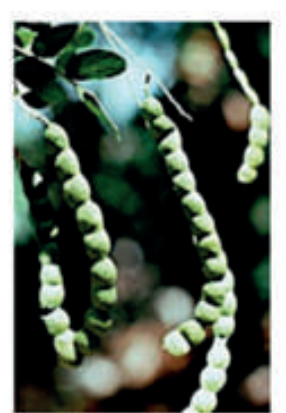

FABACEAF

Albizia pistaciifolia

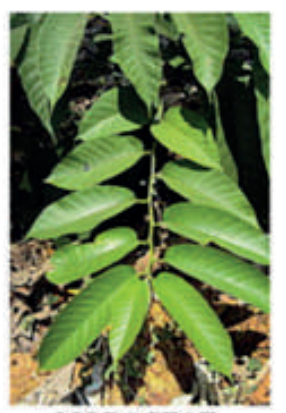

MORACEAE

Castilla elastica

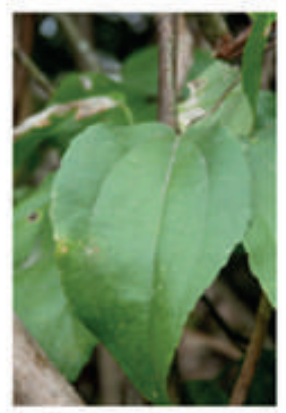

RHANNACEAE

Zizyphus thyrsiflora 


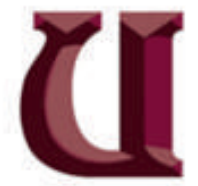

\section{IEES}

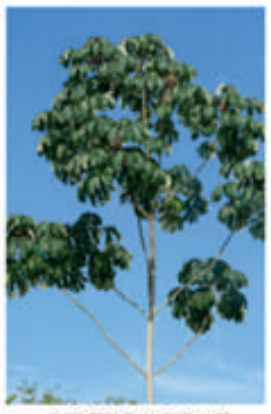

URTICACEAE

Cecropia obtusifolia

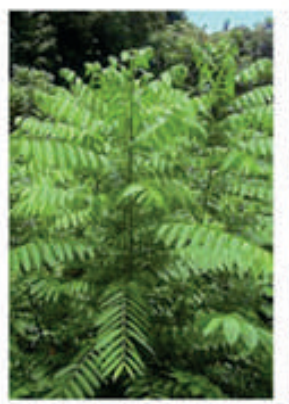

MELIACEAE

Cedrela odorata

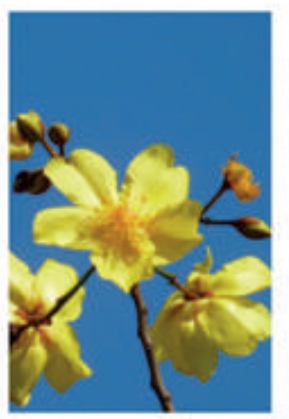

BIXACEAE

Cochlospermun vitifolium

\section{Bosque Seco Tropical Zona de Vida Silvestre \\ Parque Histórico Guayaquil}

Universidad de Especialidades Espíritu Santo

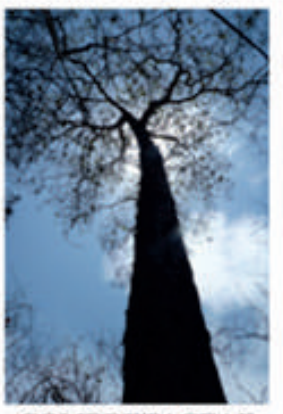

COMBRETACEAE

Terminalia valverdae

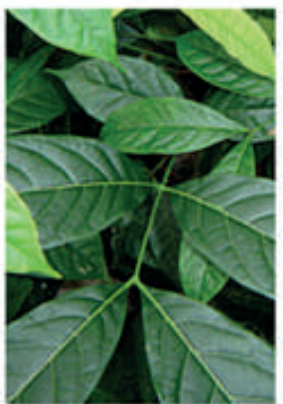

MELIACEAE

Swetenia macrophyllia

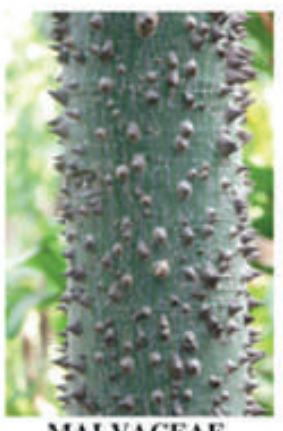

MALVACEAE

Ceiba trichistandra

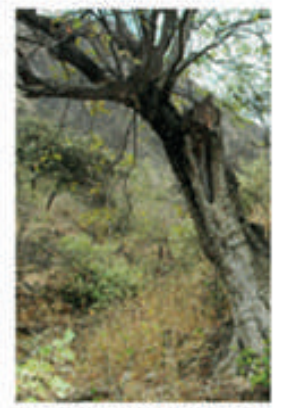

ANACARDIACEAE
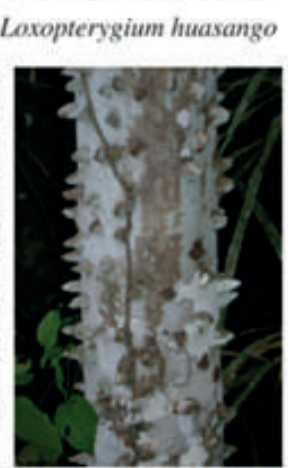

RUTACEAE

Zanthoxylum setulosum

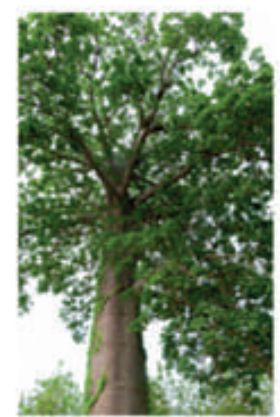

MALVACEAE

Cavanillesia platanifolia

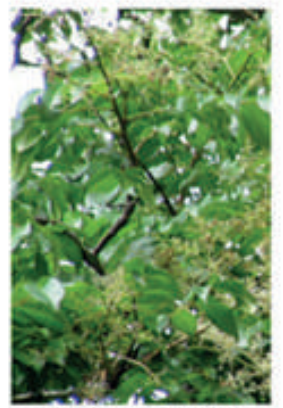

ANACARDIACEAE

Spondia mombim

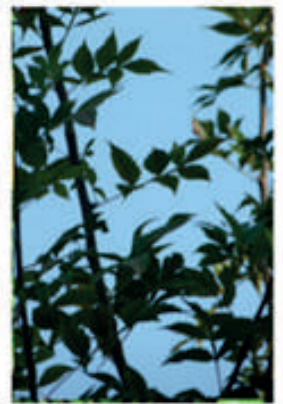

BURSERACEAE

Bursera graveolens

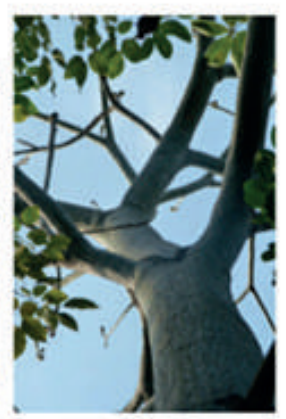

MALVACEAE

Pseudobombax millei
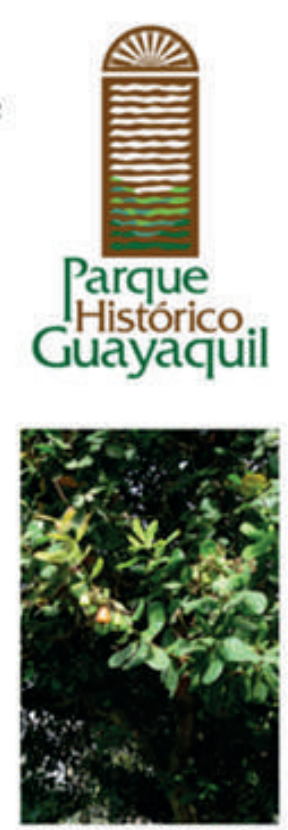

ANACARDIACEAE

Anacardium sp.

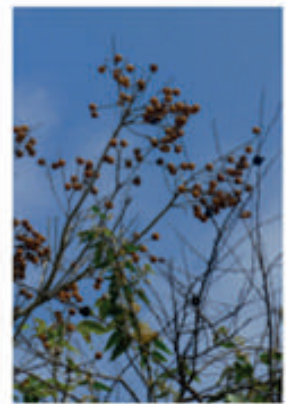

SAPINDACEAE

Sapindus saponaria

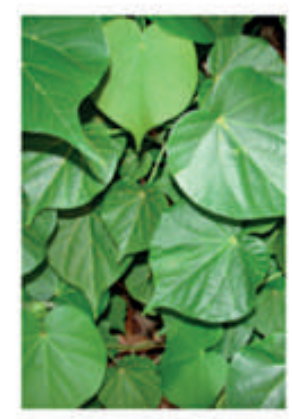

MALVACEAE.

Hibiscus tiliaceus

\section{3}

INVESTIGATIO No. 9, marzo 2017, pp. 1-27

(C) Universidad Espíritu Santo - UEES

ISSN: 1390 - 6399 


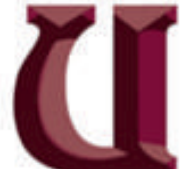

UEES

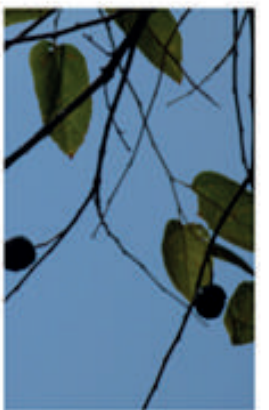

MALVACEAE

Guazuma ulmifolia

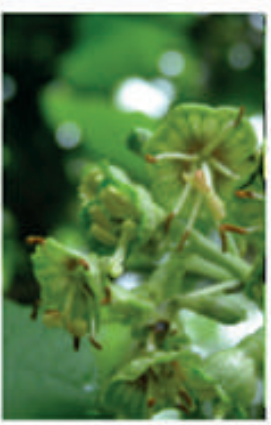

RUBIACEAE

Simira ecuadoriensis

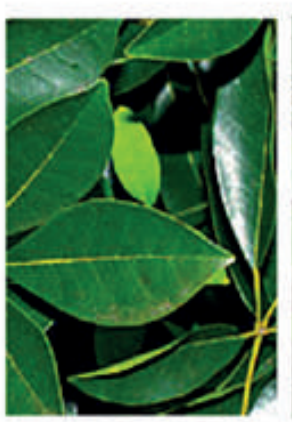

BIGNONIACEAE

Tabebuia heterophylla
Bosque Seco Tropical Zona de Vida Silvestre

Parque Histórico Guayaquil

Universidad de Especialidades Espíritu Santo

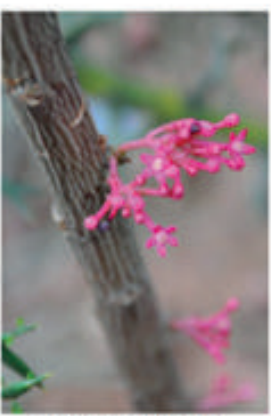

CARICACEAE

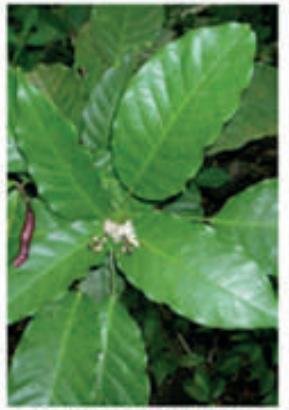

CAPPARACEAE

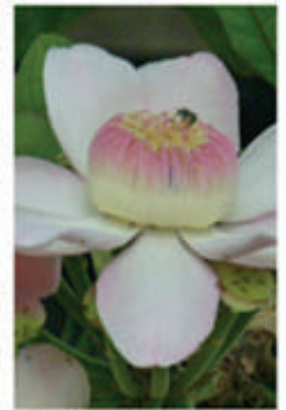

LECYTIDACEAE

Vasconcellea parviflora Capparidastnam bonifazianum Gustavia angustifolia

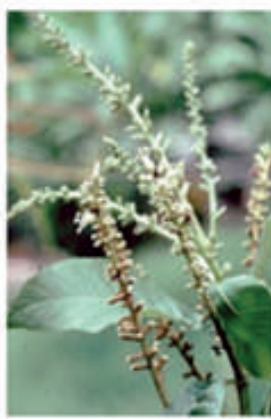

RUBLACEAE

Alseis eggersi

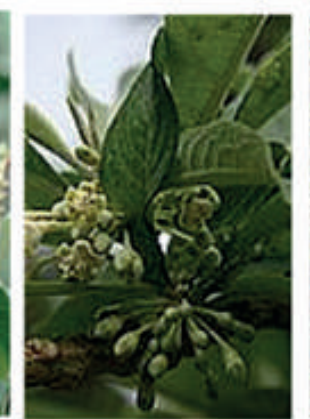

SOLANACEAE

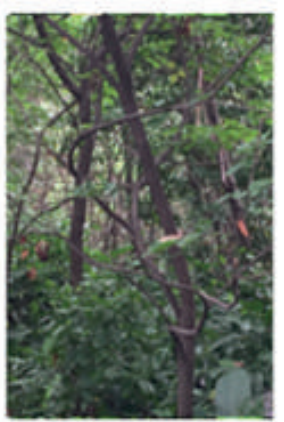

BIGNONIACEAE

Acnistus arborescens Handroanthus chrysanthus. Handroanthus chrysanthus

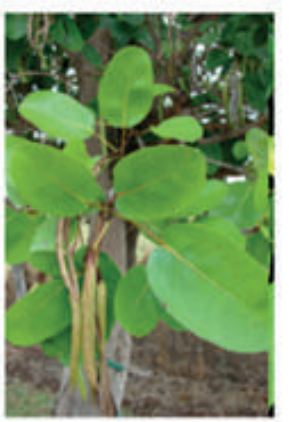

BIGNONIACEAE

Tabebuia rosea

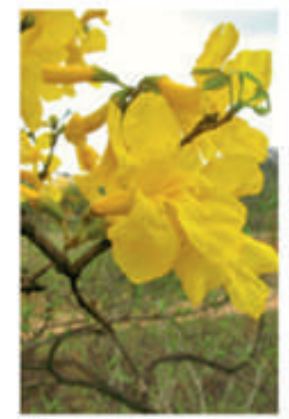

BIGNONIACEAE

Handroanthus billbergii

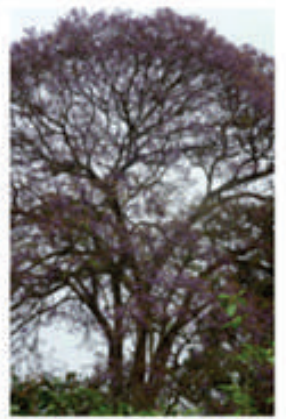

VERBENACEAE

Vitex gigantea

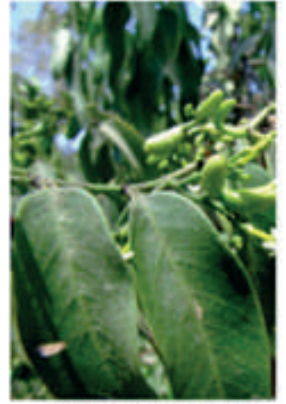

APOCYNACEAE

Vallesia glabra

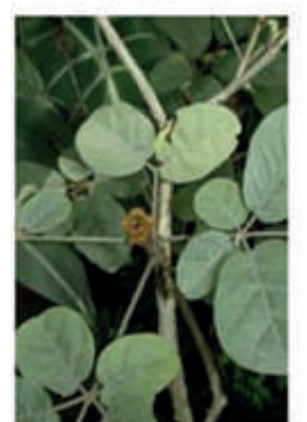

BIGNONIACEAE

ssp. pluvicola

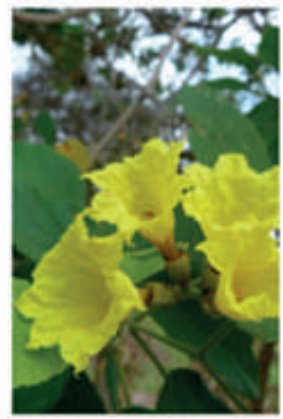

BORRAGINACEAE

Cordia lutea

\section{4}

INVESTIGATIO No. 9, marzo 2017, pp. 1-27

(C) Universidad Espíritu Santo - UEES

ISSN: 1390 - 6399 


\section{5}

\section{【1 \\ UEES}

Bosque Seco Tropical Zona de Vida Silvestre

Parque Histórico Guayaquil

Universidad de Especialidades Espíritu Santo

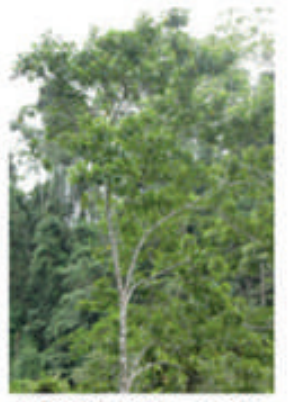

BORRAGINACEAE

Cordia alliodora

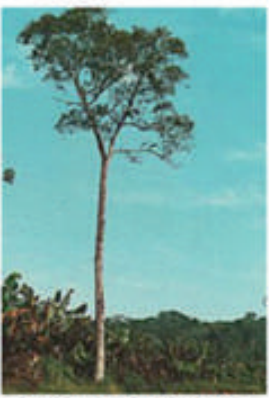

BORRAGINACEAE

Cordia macrantha

Anexo 4.

Tabla 5.

Distribución de especies por DAP y especies por altura (Orden descendente)

\begin{tabular}{|l|l|}
\hline Especie / DAP & Especie / altura \\
\hline Pseudobombax millei (Standl) A. Robyns & Pseudobombax millei (Standl) A. Robyns \\
\hline Tabebuia rosea (Bertol.) DC. & Anacardium sp. L. \\
\hline Cochlospermun vitifolium (Willd.) Spreng. & Terminalia valverdae G. \\
\hline Vismia obtusa Spruce & Phyllanthus juglandifolius (L) Wild \\
\hline Swetenia macrophyllia King & Swetenia macrophyllia King \\
\hline Vitex gigantea Kunth & Tabebuia rosea (Bertol.) DC. \\
\hline Terminalia valverdae G. & Vismia obtusa Spruce \\
\hline Ceiba trichistandra (A. Gray) Bakh. & Piscidia carthagenensis Jacq. \\
\hline Guazuma ulmifolia Lam. & Cecropia obtusifolia Bertol \\
\hline Anacardium sp. L. & Cedrela odorata L. \\
\hline
\end{tabular}




\begin{tabular}{|c|c|}
\hline Cedrela odorata $\mathrm{L}$. & Vitex gigantea Kunth \\
\hline Maclura tinctoria (L) Steud & Cochlospermun vitifolium (Willd.) Spreng. \\
\hline $\begin{array}{l}\text { Handroanthus chrysanthus ssp. pluvicola } \\
\text { (A.H. Gentry) S.O. Grose }\end{array}$ & Cordia macrantha Chodat \\
\hline Cecropia obtusifolia Bertol & Brownea ariza Benth. \\
\hline Piscidia carthagenensis Jacq. & Castilla elastica Cervantes \\
\hline Cordia macrantha Chodat & Myroxylom peruiferum $\mathrm{Lf}$ \\
\hline Zizyphus thyrsiflora Benth & Maclura tinctoria (L) Steud \\
\hline Erythrina smithiana Krukoff & Guazuma ulmifolia Lam. \\
\hline Phyllanthus juglandifolius (L) Wild & Erythrina smithiana Krukoff \\
\hline Hibiscus tiliaceus L. & Spondia mombim L. \\
\hline Loxopterygium huasango Spruce ex Engl. & $\begin{array}{l}\text { Handroanthus billbergii (Bureau \& K.Schum.) } \\
\text { S.O.Grose }\end{array}$ \\
\hline Spondia mombim L. & Gliricidia sepium (Jack.) Kunt ex Walp. \\
\hline Caesalpinia glabrata Kunth & Centrolobium ochroxylum Rose ex Rub \\
\hline Brownea ariza Benth. & $\begin{array}{l}\text { Handroanthus chrysanthus ssp. pluvicola } \\
\text { (A.H. Gentry) S.O. Grose }\end{array}$ \\
\hline Couepia subcordata Benth. Ex. Hook F. & Licaria limbosa (R\&P) Kosterm \\
\hline Erythrina velutina Wild & Cordia alliodora (R\&P) Oken Allgen \\
\hline Cordia alliodora (R\&P) Oken Allgen & Loxopterygium huasango Spruce ex Engl. \\
\hline Vallesia glabra (Cav) Link & Erythrina velutina Wild \\
\hline Myroxylom peruiferum Lf & Zanthoxylum setulosum P. Wilson \\
\hline Castilla elastica Cervantes & Alseis eggersi Stadl \\
\hline Bursera graveolens H.B.K & Browneopsis disepala (little) Klitgaard \\
\hline Cavanillesia platanifolia (Bonpl.) Kunth & Ocotea sp. \\
\hline $\begin{array}{l}\text { Handroanthus billbergii (Bureau \& K.Schum.) } \\
\text { S.O.Grose }\end{array}$ & Zizyphus thyrsiflora Benth \\
\hline Acnistus arborescens (L.) Schlecht & Cavanillesia platanifolia (Bonpl.) Kunth \\
\hline
\end{tabular}




\begin{tabular}{|l|l|}
\hline Zanthoxylum setulosum P. Wilson & Couepia subcordata Benth. Ex. Hook F. \\
\hline Gliricidia sepium (Jack.) Kunt ex Walp. & Hibiscus tiliaceus L. \\
\hline Centrolobium ochroxylum Rose ex Rub & Castilla tunu Hemsl \\
\hline Pithecellobium dulce & Brosimum alicastrum Sw. \\
\hline
\end{tabular}

